\title{
Research Paper: Modulatory Effects of Memantine on ๑) (ఎ) Neuronal Response Properties in Rat Barrel Cortex
}

\author{
Mahin Nasiri ${ }^{1}$ (D), Ayat Kaeidi ${ }^{1,2}$ (D), Iman Fatemi ${ }^{3}$ (D), Mahdieh Azin ${ }^{1,2}$ (D), Mahboobeh Bannazadeh ${ }^{1}$ (D), Mohammad Allahtavakoli ${ }^{1,2}$ (D), Ali \\ Roohbakhsh $^{4}$ (D), Ali Shamsizadeh ${ }^{1,2^{*}}$ (D) \\ 1. Physiology-Pharmacology Research Center, Research Institute of Basic Medical Sciences, Rafsanjan University of Medical Sciences, Rafsanjan, Iran. \\ 2. Department of Physiology and Pharmacology, School of Medicine, Rafsanjan University of Medical Sciences, Rafsanjan, Iran. \\ 3. Research Center of Tropical and Infectious Diseases, Kerman University of Medical Sciences, Kerman, Iran. \\ 4. Pharmaceutical Research Center, Pharmaceutical Technology Institute, Mashhad University of Medical Sciences, Mashhad, Iran
}

\begin{tabular}{|c|c|}
\hline $\begin{array}{l}\text { Use your device to scan } \\
\text { and read the article online }\end{array}$ & Ctition Nasiri, M., Kaeidi, A., Fatemi, I., Azin, M., Bannazadeh, M., \& Allahtavakoli, M., et al. (2021). Modulatory Effects \\
\hline 口it:sira & $\begin{array}{l}\text { of Memantine on Neuronal Response Properties in Rat Barrel Cortex. Basic and Clinical Neuroscience, 12(6), 721-728. http:// } \\
\text { dx.doi.org/10.32598/bcn.2021.1156.2 }\end{array}$ \\
\hline 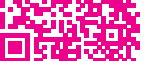 & delisttp://dx.doi.org/10.32598/bcn.2021.1156.2 \\
\hline
\end{tabular}

\section{(c) (i) (3)}

Article info:

Received: 16 May 2019

First Revision: 25 Sep 2019

Accepted: 02 Dec 2019

Available Online: 01 Nov 2021

Keywords:

Memantine, Somatosensory cortex, Electrophysiology, Rat

\section{A B S T RACT}

Introduction: Memantine as N-Methyl-D-Aspartic Acid (NMDA) receptor antagonist is used in some neurological disorders. Moreover, memantine presents modulatory effects on the somatosensory information processing in healthy subjects. This study investigated the effects of memantine on electrophysiological properties of barrel cortex neurons in male rats.

Methods: Single unit recording was used to evaluate the electrophysiological properties of barrel cortex neurons. The neural responses to the Principal Whisker (PW), Adjacent Whisker (AW), and combined displacement of two whiskers [20 ms Inter-Stimulus Intervals (ISIs)] were recorded before and 2 hours after memantine gavage $(10 \mathrm{mg} / \mathrm{kg})$. A Condition Test Ratio (CTR) was calculated for assessing inhibitory interactions.

Results: Two hours after memantine gavage, neuronal ON and OFF responses to PW deflection were decreased. Furthermore, CTR for both ON and OFF responses was decreased following memantine administration.

Conclusion: The current study demonstrated that memantine modified neural response properties in the rat barrel cortex.

* Corresponding Author:

Ali Shamsizadeh, PhD.

Address: Physiology-Pharmacology Research Center, Research Institute of Basic Medical Sciences, Rafsanjan University of Medical Sciences,

Rafsanjan, Iran.

Tel: +98 (34) 34339660

E-mail: ashamsi@rums.ac.ir; alishamsy@gmail.com 


\section{Highlights}

- Memantine modulated excitatory receptive fields in the rat somatosensory cortex.

- Memantine decreased integrative receptive fields in rat somatosensory cortex.

\section{Plain Language Summary}

As an NMDA receptor antagonist, memantine is used to treat moderate to severe Alzheimer's disease. Memantine has beneficial effects on cognition, mood, and perform daily activities. However, the current study results suggested that memantine may affect information processing in the somatosensory system. This should be considered for future research in the clinic.

\section{Introduction}

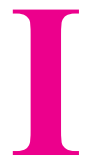

$\mathrm{t}$ is widely accepted that NMDA $(\mathrm{N}$ Methyl-D-Aspartic Acid) receptors play a critical role in learning, memory, and plastic changes in the nervous system (Bradley et al., 2017). Numerous reports demonstrate that NMDA receptor dysfunction in many neurological diseases, such as Alzheimer's, Parkinson's, Huntington's, Stroke, and nerve pain (Kimura, Namekata, Guo, Harada, \& Harada, 2017; Makhaeva et al., 2015). In clinical conditions, the blockers of NMDA receptors, such as Ketamine, Amantadine, and Dextromethorphan are used to alleviate harmful effects of calcium and the over-activation of NMDA receptors in neurological diseases (Bowers, McAllister, Ray, \& Heitz, 2017; Martinak, Bolis, Black, Fargason, \& Birur, 2017). Memantine is a non-competitive antagonist of NMDA receptors discovered in the 1960s. Lately (in the 1980s), it was used to treat attention and episodic memory disturbances in Parkinson's disease and dementia. In 2003, it was also prescribed to treat cognitive and behavioral dysfunctions in Alzheimer's disease (Johnson \& Kotermanski, 2006). Dizziness, headache, constipation, somnolence were reported as common side effects of memantine and adverse events are usually mild to moderate in severity (van Marum, 2009). Dinse et al. reported that memantine might modulate somatosensory information processing in healthy humans (Dinse, Ragert, Pleger, Schwenkreis, \& Tegenthoff, 2003).

With the barrel cortex as its most prominent structure, the rodent whisker system has a precise plan of functional organization, development, and plasticity. These features make the whisker to the barrel system a good experimental model for studying the somatosensory system (Stuttgen \& Schwarz, 2018).
This study evaluated the effects of memantine on the electrophysiological properties of neurons in the somatosensory cortex by using a whisker barrel model in rats.

\section{Methods}

\section{Animals}

Thirty male Wistar rats (weighing 200-250 g) were used in this study. They were kept in standard cages, under a 12/12 hour light-dark cycle and a Mean \pm SD temperature of $23 \pm 0.2^{\circ} \mathrm{C}$, with free access to food and water. All experimental procedures were carried out per the guidelines for the care and use of laboratory animals in the Rafsanjan University of Medical Sciences and the European Communities Council Directive 2010/63/EU.

The rats were anesthetized using urethane $(1.5 \mathrm{~g} / \mathrm{kg}$, intraperitoneal). Then, a gavage tube was transferred through the rat's mouth into its stomach. It was fixed after confirming the accuracy of its location in the stomach Next, the rat was placed into a Stereotaxic device (Stoelting, USA) and rectal temperature was maintained at 37 ${ }^{\circ} \mathrm{C}$ by a servo-controlled heating blanket (Harvard Apparatus, Eden Bridge, UK). The level of consciousness was evaluated through monitoring the tail and hind limb reflexes, regularity of respiration (80-100 breaths/min), the absence of spontaneous movements and the absence of overt whisking or whisker tremor. If necessary, $10 \%$ of the initial dose of urethane was injected to maintain the appropriate level of anesthesia (Shamsizadeh et al., 2007).

The recording device was a tungsten microelectrode (FHC, Bowdoinham, ME, U.S; (tip impedance 1-1.2 MOhms at $1000 \mathrm{~Hz}, 10 \mathrm{nA})$ ) attached to the micromanipulator (WPI Company). This micromanipulator was fixed on a Stereotaxic apparatus (Stoelting, U.S.) to modulate the electrode's location and height. Recorded 
potentials from nerve cells were amplified and digitized by the Electromodule system (Electrophysiology recording system Science Beam Inst, Tehran, Iran).

The recorded signals were imported to eSorter (Spike Sorting Software toolbox, www.ScienceBeam.com), and spike potentials peak and valley amplitude features were extracted and classified by k-, means clustering offline. The $\mathrm{ON}$ and OFF responses were detected by further analysis of spikes using eSpike software (spike analyzing software toolbox, www.ScienceBeam.com).

Whiskers displacement was performed by two tiny speakers (Electromodule, Science Beam Inst) (Motaghi, Sheibani, Farazifard, \& Joneidi, 2006; Shamsizadeh et al., 2007). A thin glass tube with an internal diameter of $0.69 \mathrm{~mm}$ was attached to the center of each speaker. The Principal Whisker (PW) and Adjacent Whisker (AW) were cut $10 \mathrm{~mm}$ from the base and inserted inside the tube. These speakers produced a ramp and hold movement with the following characteristics. Whiskers displacement time to reach new position was $5 \mathrm{~ms}$. The duration of staying in the new position was $200 \mathrm{~ms}$. Whisker returning time from new position to its initial position was $5 \mathrm{~ms}$. The displacement amplitude was 500 micrometers. The numbers of displacements equaled 40 .

Extracellular single-unit recording: After anesthetizing and placing the rat in a Stereotaxic apparatus, craniotomy of the right primary somatosensory cortex (stereotaxic coordinates: $3.3 \mathrm{~mm}$ posterior to bregma \& 4.85-5.0 mm lateral from the midline) was performed and the microelectrode was put in the cortex. To ensure the place of a microelectrode in the barrel cortex as well as to detect the PW, firstly, microelectrode inserted at a depth of 500-800 micrometers from the surface of the skull then, PW was stimulated and the latency of neuronal response was measured. In all experiments, the response latency was below $10 \mathrm{~ms}$. After that, the cortex was covered with $3 \%$ agar solution in saline and the microelectrode was moved back to superficial layers of cortex (depth of 200-400 micrometers). One of the neighboring whiskers (in same row with PW) with the least neural responses was considered as AW. The rest of rat whiskers were shortened by a scissor to avoid missstimulations (Salaria, Shamsizadeh, Noorbakhsh, Ayoobi, Sheibani, \& Oryan, 2017).

Following whiskers displacement, two neuronal responses can be seen, known as $\mathrm{ON}$ and OFF responses. ON response is produced when the whisker moves from its initial condition to the new one, and OFF response is produced when the whisker returns to its initial state (Simons \& Land, 1987). The whisker displacement direction was ventral for $\mathrm{ON}$ and dorsal for OFF responses.
To evaluate the excitatory receptive field, PW and AW were moved separately. To evaluate the paired whisker interactions, the AW displaced at $20 \mathrm{~ms}$ before the displacement of PW. Each of these protocols was repeated 40 times with a frequency of $1 \mathrm{~Hz}$, and the corresponding responses saved in separate files.

Peristimulus Time Histograms (PSTHs) were produced in response to each of the nine deflection patterns. Magnitudes of the ON and OFF responses were calculated by counting the number of spikes from 5 to $35 \mathrm{~ms}$ after the onset of each movement. The effect of the AW deflection on the unit's response to the PW deflection was quantified using a Condition-Test Ratio (CTR). CTR is calculated as follows, the magnitude of response to PW in paired deflection paradigm with AW (recorded with different delays between AW \& PW stimulation)/the magnitude of response to deflection of PW plus magnitude of response to deflection of AW when they deflected alone. If the CTR is less than 1, it shows the inhibitory effect of AW on the neuronal response to PW. If it is more than 1 , it is considered a facilitatory effect of AW on the neuronal response to PW (Ego-Stengel, Souza, Jacob, \& Shulz, 2005; Simons \& Carvell, 1989).

The rat was gavaged with memantine $(E b i x a \AA, H$. Lundbeck A/S, Denmark) at a dose of $10 \mathrm{mg} / \mathrm{kg}$. The drug was solved in normal saline $(1 \mathrm{~mL})$. Previous reports demonstrated that $10 \mathrm{mg} / \mathrm{kg}$ of memantine could prevent imipramine-induced cognitive impairments in rats (Demontis, Falconi, Canu, \& Serra, 2015). Lee et al. reported that the maximum plasma concentration of memantine would reach around $2 \mathrm{~h}$ after its gavage $(10 \mathrm{mg} /$ $\mathrm{kg}$ ) (Lee et al., 2016). Then, electrophysiological recordings were done before and $2 \mathrm{~h}$ after memantine gavage.

Paired Samples t-test was used to compare CTR, ON, and OFF responses before and after memantine gavage. The obtained data were presented as Mean \pm SEM. Moreover, SPSS and Excel software were used for data analysis.

\section{Results}

A population PSTHs of neuronal responses to PW deflection before and after administration of memantine are shown in Figure 1 and 2 shows the modulation of neuronal responses to PW deflection in superficial layers of the barrel cortex by memantine. We measured neuronal $\mathrm{ON}$ and $\mathrm{OFF}$ responses to $\mathrm{PW}$ stimulation before and after drug administration (see Methods). Statistical analysis showed that both neuronal ON $\left(\mathrm{t}_{18}=3.30\right.$, $\mathrm{P}=0.008)$ and OFF $\left(\mathrm{t}_{20}=2.65, \mathrm{P}=0.015\right)$ response magnitudes to $\mathrm{PW}$ deflection were decreased following the 

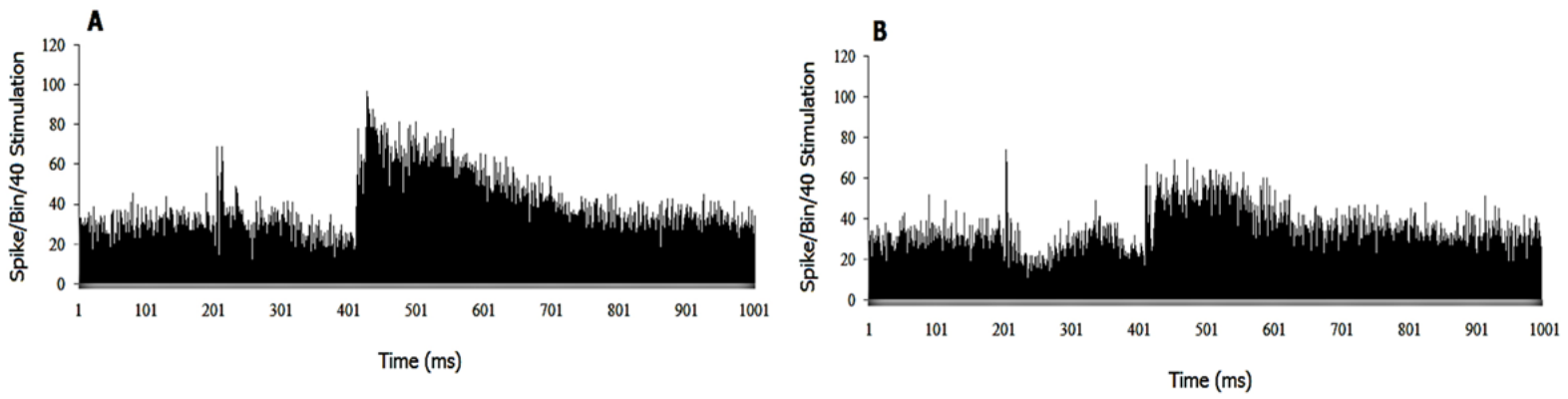

NEUR SCIENCE

Figure 1. Population Peristimulus Time Histograms (PSTHs) of neuronal responses to Principal Whisker (PW) deflection before (A) and after (B) the administration of memantine

Data were collected from 22 neurons. Bin size is $1 \mathrm{~ms}$.

administration of memantine. The gavage of memantine had no effect on the $\mathrm{ON}$ and $\mathrm{OFF}$ responses to AW stimulation ( $\mathrm{ON}$ responses $\mathrm{t}_{21}=0.55, \mathrm{P}=0.588 \& \mathrm{OFF}$ responses $\mathrm{t}_{21}=1.59, \mathrm{P}=0.125$ ) (Figure 3).

Paired whisker deflection paradigm is usually used to study the inhibitory receptive fields. In this paradigm, AW was deflected before PW at $20 \mathrm{~ms}$ Inter-Stimulus Intervals (ISIs). Results were quantified by calculating a conditioning-test ratio. Paired Samples t-test analysis demonstrated that acute oral administration of the memantine decreased CTR ratio for $\mathrm{ON}\left(\mathrm{t}_{21}=2.32, \mathrm{P}=0.03\right)$ and $\mathrm{OFF}$ $\left(\mathrm{t}_{(20)}=3, \mathrm{P}=0.006\right)$ responses (Figure 4$)$. In summary, these

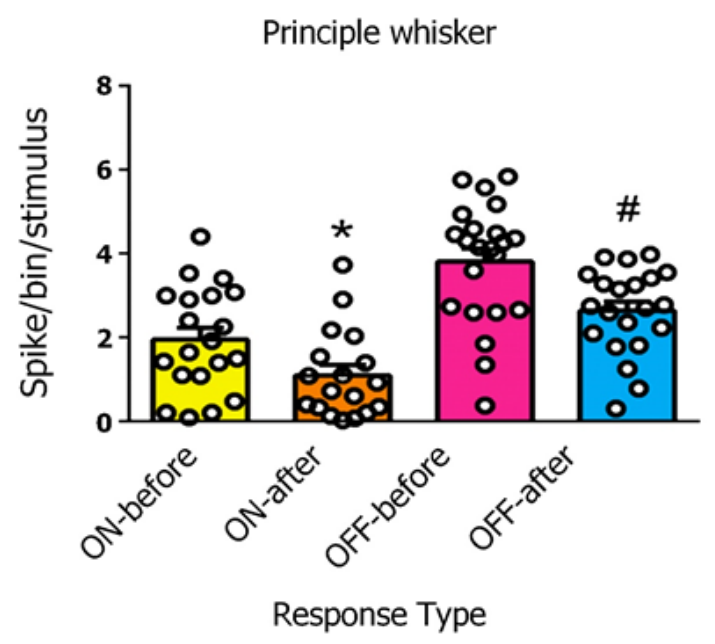

NEUR:SCIENCE

Figure 2. Neuronal ON and OFF responses to the deflection of PW before and after gavage of the memantine

*Versus before drug administration for ON responses, $\mathrm{P}=0.008$; "Versus before drug administration for OFF responses; $\mathrm{P}=0.015$. Data are expressed as Mean \pm SEM. The bin size was set at $1 \mathrm{~ms}$. data showed that memantine could modulate neuronal response properties in the somatosensory cortex.

\section{Discussion}

The present study investigated the acute effects of memantine gavage on rats' somatosensory system through electrophysiological (neuronal responses to natural stimuli using the vibrissae pathway model) approach. The obtained results suggested that acute gavage of the memantine modulated both excitatory and inhibitory receptive fields in the barrel cortex.

When rats explore the environment, they touch objects with whiskers. The mechanical receptors connected to the

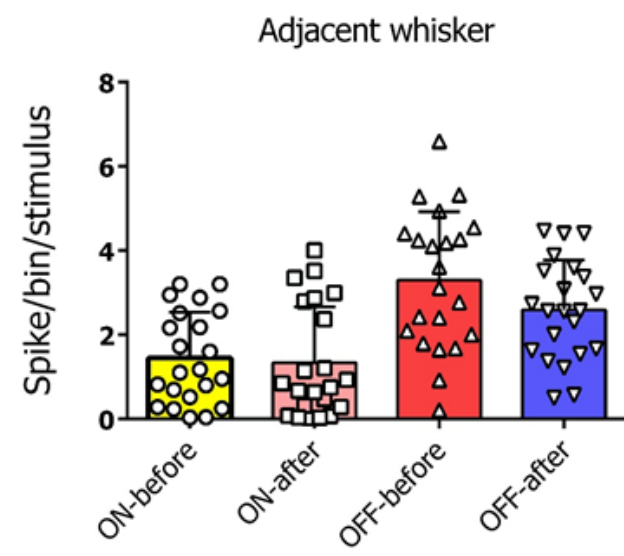

Response Type

NEUR SCIENCE

Figure 3. Neuronal ON and OFF responses to the deflection of $\mathrm{AW}$ before and after gavage of the memantine

Responses were not significantly different before and after the administration of memantine. Data are expressed as Mean \pm SEM. The bin size was set at $1 \mathrm{~ms}$. 


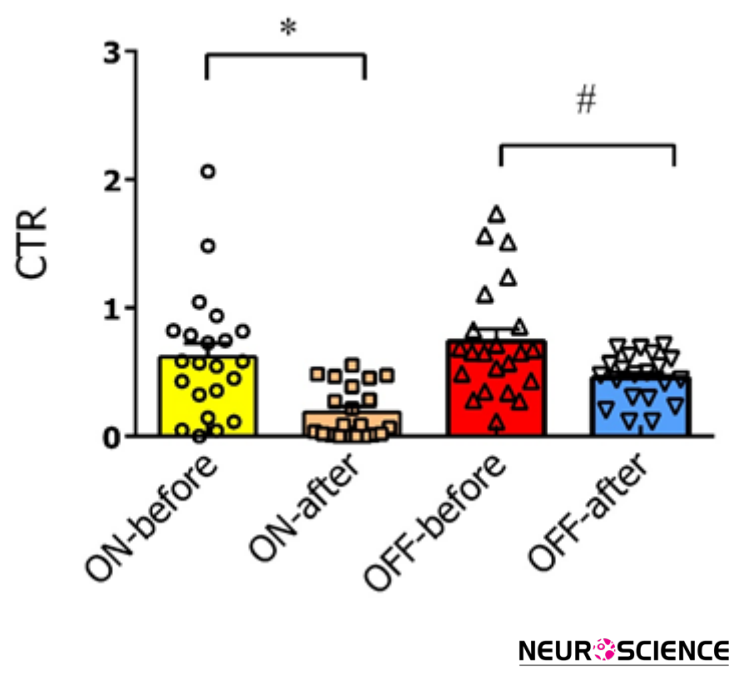

Figure 4. Condition Test Ratios (CTR) for ON and OFF responses before and after gavage of the memantine

A CTR of $>1$ indicates facilitation of the response to deflection of the first whisker, whereas a CTR of $<1$ denotes suppression of the response to deflection of the second whisker. Data are expressed as Mean $\pm S E M$; *Versus corresponding time point before drug administration $(\mathrm{P}=0.03)$, "Versus corresponding time point before drug administration $(\mathrm{P}=0.006)$

whiskers transmit the information from the environment to the primary somatosensory cortex (mostly on layer 4 , i.e., called barrel in rodents) that have both excitatory and inhibitory receptive fields (Fox, Wright, Wallace, \& Glazewski, 2003; Wright \& Fox, 2010). Then, the sensory signal is projected to superficial layers (2 and 3 ) of the corresponding column. After that, they are launched to the surface layers of adjacent columns (Nussbaumer \& Van der Loos, 1985). This signal circuit plays an essential role in recognizing and processing $3 \mathrm{D}$ objects in the brain (Armstrong-James \& Fox, 1987). The result of this study that demonstrated the modulatory effects of memantine on excitatory and inhibitory receptive fields in the superficial layers of the barrel cortex will naturally change the pattern of information processing.

In line with these results, other investigators also reported that NMDA agonists and antagonists could modulate neuronal activities in the cerebral cortex. Armstrong-James et al. reported that administration of 6.7-dinitroquinoxaline-2,3-dione (an NMDA receptor antagonist) could modulate the neuronal activities in the barrel cortex (Armstrong-James, Welker, \& Callahan, 1993). In an in vivo study, infused d-isomer of 2-amino-5 phosphonopentanoic acid (D-AP5) (a NMDA receptor antagonists) over the barrel cortex recorded from cells in layer II-III of barrel cortex following the induction of specific pattern of neural plasticity. They concluded that cortical
NMDARs are necessary for experience-dependent plasticity in the barrel cortex (Rema, Armstrong-James, \& Ebner, 1998). In another interesting study, Dinse et al. studied the effect of memantine on two-point discrimination ability in healthy people through recording somatosensory field potentials. They reported the modulatory effect of memantine on this ability (Dinse et al., 2003). The results of our study with previous studies conclude that memantine may have a deleterious effect on somatosensory information processing both in rats and humans.

However, some reports are supporting minor behavioral effects of the meantime. Pringle et al. examined the effects of memantine on emotional processing in 32 healthy young volunteers. They concluded that acute memantine administration has limited sensory information processing in healthy individuals (Pringle Parsons, Cowen, McTavish, Cowen, \& Harmer, 2012). On the other hand, chronic treatment of adult rats with memantine (30 $\mathrm{mg} / \mathrm{kg} /$ day for 8 weeks) had beneficial effects on learning abilities in the Morris water maze task (Barnes, Danysz, $\&$ Parsons, 1996). A growing number of studies suggest the credible efficacy of memantine in treating some neurodegenerative disorders, like Alzheimer's disease (Kishi, Matsunaga, Oya, Nomura, Ikuta, \& Iwata, 2017).

\section{Conclusion}

Our results indicates that a therapeutic dose of memantine presents modulatory effects on somatosensory information processing in rats. Thus, further studies using animal models of neurodegenerative disorders have to be conducted to understand the possible side effects of memantine on the somatosensory system in disease conditions.

\section{Ethical Considerations}

\section{Compliance with ethical guidelines}

The study was approved by the Ethics Committee of Rafsanjan University of Medical Sciences (Code: IR.RUMS.REC.1396.76)

\section{Funding}

This research was supported by the Rafsanjan University of Medical Sciences (No. 20/1065).

\section{Authors' contributions}

Conceptualization and supervision: Ali Roohbakhsh and Ali Shamsizadeh; Methodology: Iman Fatemi and Mohammad Allahtavakoli; Investigation, writing - origi- 
nal draft, writing - review \& editing: All authors; Data collection: Mahin Nasiri and Mahboobeh Bannazadeh; Data analysis: Ayat Kaeidi and Ali Shamsizadeh; Funding acquisition and resources: Ali Shamsizadeh.

\section{Conflict of interest}

The authors declared no conflict of interest.

\section{Acknowledgments}

The authors acknowledge the Research Councils of Rafsanjan Universities of Medical Sciences for their support.

\section{Refrences}

Armstrong James, M., Welker, E., \& Callahan, C. A. (1993). The contribution of NMDA and non-NMDA receptors to fast and slow transmission of sensory information in the rat SI barrel cortex. Journal of Neuroscience, 13(5), 2149-60. [DOI:10.1523/JNEUROSCI.13-05-02149.1993] [PMID] [PMCID]

Armstrong James, M., \& Fox, K. (1987). Spatiotemporal convergence and divergence in the rat S1 "barrel" cortex. Journal of Comparative Neurology, 263(2), 265-81. [DOI:10.1002/cne.902630209] [PMID]

Barnes, C. A., Danysz, W., \& Parsons, C. G. (1996). Effects of the uncompetitive NMDA receptor antagonist memantine on hippocampal long-term potentiation, short-term exploratory modulation and spatial memory in awake, freely moving rats. European Journal of Neuroscience, 8(3), 565-71. [DOI:10.1111/j.1460-9568.1996.tb01241.x] [PMID]

Bowers, K. J., McAllister, K. B., Ray, M., \& Heitz, C. (2017). Ketamine as an adjunct to opioids for acute pain in the emergency department: A randomized controlled trial. Academic Emergecy Medicine, 24(6), 676-85. [DOI:10.1111/acem.13172] [PMID]

Bradley, R. M., Mardian, E. B., Bloemberg, D., Aristizabal Henao, J. J., Mitchell, A. S., \& Marvyn, P. M., et al. (2017). Mice deficient in lysophosphatidic acid acyltransferase delta (lpaatdelta)/ acylglycerophosphate acyltransferase 4 (agpat4) have impaired learning and memory. Molecular and Cellular Biology, 37(22). [DOI:10.1128/MCB.00245-17] [PMID] [PMCID]

Demontis, F., Falconi, M., Canu, D., \& Serra, G. (2015). Memantine prevents "bipolar-like" behavior induced by chronic treatment with imipramine in rats. European Journal of Pharmacology, 752, 4954. [DOI:10.1016/j.ejphar.2015.01.041] [PMID]

Dinse, H. R., Ragert, P., Pleger, B., Schwenkreis, P., \& Tegenthoff, M. (2003). Pharmacological modulation of perceptual learning and associated cortical reorganization. Science, 301(5629), 91-94. [DOI:10.1126/science.1085423] [PMID]

Ego-Stengel, V., Souza, T. M. E., Jacob, V., \& Shulz, D. E. (2005). Spatiotemporal characteristics of neuronal sensory integration in the barrel cortex of the rat. Journal of neurophysiology, 93(3), 1450-1467. [DOI:10.1152/jn.00912.2004] [PMID]

Fox, K., Wright, N., Wallace, H., \& Glazewski, S. (2003). The origin of cortical surround receptive fields studied in the barrel cortex.
Journal of Neuroscience, 23(23), 8380-91. [DOI:10.1523/JNEUROSCI.23-23-08380.2003] [PMID] [PMCID]

Johnson, J. W., \& Kotermanski, S. E. (2006). Mechanism of action of memantine. Current Opinion in Pharmacology, 6(1), 61-7. [DOI:10.1016/j.coph.2005.09.007] [PMID]

Kimura, A., Namekata, K., Guo, X., Harada, C., \& Harada, T. (2017). Dock3-NMDA receptor interaction as a target for glaucoma therapy. Histology $\mathcal{E}$ Histopathology, 32(3), 215-21. [DOI:10.14670/HH-11-820] [PMID]

Kishi, T., Matsunaga, S., Oya, K., Nomura, I., Ikuta, T., \& Iwata, N. (2017). Memantine for alzheimer's disease: An updated systematic review and meta-analysis. Journal of Alzheimer's Disease, 60(2), 401-25. [DOI:10.3233/JAD-170424] [PMID]

Lee, S. H., Kim, S. H., Noh, Y. H., Choi, B. M., Noh, G. J., \& Park, W. D., et al. (2016). Pharmacokinetics of memantine after a single and multiple dose of oral and patch administration in rats. Basic E Clinical Pharmacology E Toxicology, 118(2), 122-7. [DOI:10.1111/bcpt.12479] [PMID]

Makhaeva, G. F., Lushchekina, S. V., Boltneva, N. P., Sokolov V. B., Grigoriev, V. V., \& Serebryakova, O. G., et al. (2015) Conjugates of gamma-carbolines and phenothiazine as new selective inhibitors of butyrylcholinesterase and blockers of NMDA receptors for alzheimer disease. Scientific Reports, 5 13164. [DOI:10.1038/srep13164] [PMID] [PMCID]

Martinak, B., Bolis, R. A., Black, J. R., Fargason, R. E., \& Birur, B. (2017). Dextromethorphan in cough syrup: The poor man's psychosis. Psychopharmacol Bull, 47(4), 59-3. [PMID] [PMCID]

Motaghi, S., Sheibani, V., Farazifard, R., \& Joneidi, H. (2006). Electrical stimulation of locus coeruleus strengthens the surround inhibition in layer V barrel cortex in rat. Neuroscience Letters, 401(3), 280-4. [DOI:10.1016/j.neulet.2006.03.034] [PMID]

Nussbaumer, J. C., \& Van der Loos, H. (1985). An electrophysiological and anatomical study of projections to the mouse cortical barrelfield and its surroundings. Journal of Neurophysiology, 53(3), 686-98. [DOI:10.1152/jn.1985.53.3.686] [PMID]

Pringle, A., Parsons, E., Cowen, L. G., McTavish, S. F., Cowen, P. J., \& Harmer, C. J. (2012). Using an experimental medicine model to understand the antidepressant potential of the N-Methyl-D-aspartic acid (NMDA) receptor antagonist memantine. Journal of Psychopharmacology, 26(11), 1417-23. [DOI:10.1177/0269881112446535] [PMID] [PMCID]

Rema, V., Armstrong-James, M., \& Ebner, F. (1998). Experiencedependent plasticity of adult rat S1 cortex requires local NMDA receptor activation. Journal of Neuroscience, 18(23), 10196-206. [DOI:10.1523/JNEUROSCI.18-23-10196.1998] [PMID] [PMCID]

Salaria, E., Shamsizadeh, A., Noorbakhsh, S. M., Ayoobi, F. Sheibani, V., \& Oryan, S. (2017). Effects of achillea millefolium aqueous extract on electrophysiological properties of rat barrel cortex neurons. Jundishapur Journal of Natural Pharmaceutical, 12(4), e37758. [DOI:10.5812/jjnpp.37758]

Shamsizadeh, A., Sheibani, V., Arabzadeh, S., Afarinesh, M R., Farazifard, R., \& Noorbakhsh, S. M., et al. (2007). Single whisker experience started on postnatal days 0,5 or 8 changes temporal characteristics of response integration in layers IV and $\mathrm{V}$ of rat barrel cortex neurons. Brain Research Bulletin 74(1), 29-36. [DOI:10.1016/j.brainresbull.2007.04.010] [PMID] 
Simons, D. J., \& Carvell, G. E. (1989). Thalamocortical response transformation in the rat vibrissa/barrel system. Journal of Neurophysiology, 61(2), 311-330. [DOI:10.1152/ jn.1989.61.2.311] [PMID]

Simons, D. J., \& Land, P. W. (1987). Early experience of tactile stimulation influences organization of somatic sensory cortex. Nature, 326(6114), 694-7. [DOI:10.1038/326694a0] [PMID]

Stuttgen, M. C., \& Schwarz, C. (2018). Barrel cortex: What is it good for? Neuroscience, 368, 3-16. [DOI:10.1016/j.neuroscience.2017.05.009] [PMID]

van Marum, R. J. (2009). Update on the use of memantine in alzheimer's disease. Neuropsychiatric Disease and Treatment, 5, 237-47. [DOI:10.2147/NDT.S4048] [PMID] [PMCID]

Wright, N., \& Fox, K. (2010). Origins of cortical layer V surround receptive fields in the rat barrel cortex. Journal of Neurophysiology, 103(2), 709-24. [DOI:10.1152/jn.00560.2009] [PMID] [PMCID] 
This Page Intentionally Left Blank 